\title{
High-risk Neuroblastoma Predisposing to Osteomyelitis: A CaseStudy
}

\author{
Vamsi Reddy ${ }^{1 *}$, Clive Miranda ${ }^{2 *}$, Nitish Sood ${ }^{1}$ and Benjamin Sookhoo $\mathrm{D}^{1}$ \\ ${ }^{1}$ Department of Orthopaedic Surgery, Georgia \\ ${ }^{2}$ Midwestern University, Arizona College of Osteopathic Medicine, Georgia
}

ISSN: 2578-0379

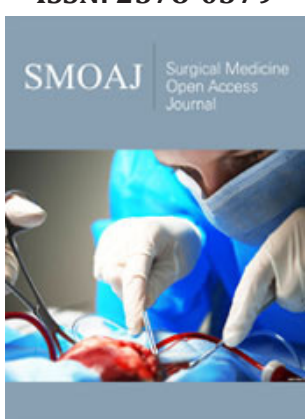

*Corresponding author: Vamsi R,Department of Orthopaedic Surgery, GeorgiaandClive M, Midwestern University, Arizona College of Osteopathic Medicine, Georgia

*Clive Miranda, Midwestern University, Arizona College of Osteopathic Medicine, Georgia

Submission: 淟 August 14, 2020

Published: 制September 15, 2020

Volume 3 - Issue 4

How to cite this article: Vamsi Reddy, Clive Miranda, Nitish S, Benjamin DS. High-risk Neuroblastoma Predisposing to Osteomyelitis: A CaseStudy. Surg Med Open Acc J. 3(4). SMOAJ. 000570. 2020. DOI: 10.31031/SMOAJ.2020.03.000570

Copyright@ Vamsi Reddy, This article is distributed under the terms of the Creative Commons Attribution 4.0 International License, which permits unrestricted use and redistribution provided that the original author and source are credited.

\begin{abstract}
Neuroblastomas are rare extracranial tumors of the pediatric population arising from cells of the embryological sympathetic nervous system. These malignancies most commonly occur in the abdomen, but other sites include the chest, neck, and pelvis with a predisposition for lymphatic and hematogenous spread. Metastasis to the bone is a poor prognostic indicator, requiring surgical excision and other extensive medical management. Staging, location, and histopathology of neuroblastomas are therefore important criteria that help stratify risk and direct treatment. We present a case report of a pediatric male with a history of metastatic neuroblastoma in remission for 3 years who was found to have a relapse of his malignancy in the bone. Further investigation yielded concomitant osteomyelitis at the recurrence site. This case illustrates the importance to evaluate for coexisting infectious processes in the setting of malignancy.
\end{abstract}

Keywords: Infection; Neuroblastoma; Oncology; Osteomyelitis; Pediatric

\section{Background}

Neuroblastomas are malignant tumors of neural crest cells and are the most common extracranial pediatric tumor in patients less than one year of age [1,2]. Around 700 new cases are identified in the United States each year and $90 \%$ of diagnosed neuroblastomas worldwide are diagnosed before 6 years of age with no strong evidence for discrepancies between sex or ethnic groups [3]. These tumors are most frequently found within the adrenal gland but can also be located in sympathetic ganglia of the retroperitoneum, mediastinum, neck or pelvis presenting locally with signs of mass effect or paraneoplastic syndromes [2]. Prognosis of nephroblastoma's is highly variable at different ages given the vast heterogeneity in stage, tumor type, and anatomic location seen in clinical cases. Low to intermediate risk tumors have much higher 5-year survival rates (90\%) than high-risk tumors do (40\% - 50\%) [2,4-6]. The determinants of higher risk include older age at diagnosis ( $>15$ months), diagnosis at later stages, and the presence of biological markers such as v-myc or N-myc [1,6-9].

There are several congenital anomalies that may co-occur in neuroblastoma patients. It is therefore critical to study cases of high-risk neuroblastomas with associated co-morbidities to help clinicians create a more informed plan of care and increase survival rates. Currently, documented co-morbidities are almost exclusively genetic in nature: von Recklinghausen disease, Beckwith-Wiedemann syndrome, Hirschsprung disease, and DiGeorge syndrome [10]. There have been no studies, however, that have demonstrated an association of comorbid infectious sequelae in neuroblastoma patients. Here, we present possibly the first reported case of neuroblastoma potentially predisposing to osteomyelitis at the same location.

\section{Case Report}

A 2-year-old boy was transferred from a regional medical center and presented with an abdominal mass, daily fevers $\left(101^{\circ} \mathrm{F}-102^{\circ} \mathrm{F}\right)$, and abdominal distension for two to three months. Over the previous week, he presented with fussiness, decreased oral intake, decreased urinary output, and dark loose stools for about a day upon admission. The mass on his left groin was biopsied, revealing small blue round cells, initially indicative of neuroblastoma. Abdominal CT scan revealed a mass consistent with Wilms tumor on his left 
kidney, retroperitoneal lymphadenopathy, and bilateral pelvic and inguinal lymphadenopathy. Subsequently, a double lumen port was placed, and a bone marrow biopsy was performed revealing metastatic neuroblastoma. An MIBG (metaiodobenzylguanidine) scan showed $>50 \%$ abdominal soft tissue involvement as well as multifocal osseous - soft tissue involvement. Ultimately, he was diagnosed with high-risk stage IV metastatic neuroblastoma with N-MYC proto-oncogene protein amplification with a large left adrenal primary with bone marrow and multiple bony metastases (Curie score 12 at diagnosis). Daily recurrent fevers along with multiple blood cultures showing no growth prompted prophylactic antibiotic administration including cefepime, ceftriaxone, zosyn, and vancomycin. Antineoplastic drugs Cytoxan and Topotecan were prescribed. Total parenteral nutrition was utilized for one and a half weeks to resolve poor oral intake. A total of four blood transfusions over seven days were given and a platelet transfusion was given the day of discharge, two weeks after admission [11].

After the initial discharge, the patient underwent induction therapy cycle 1 with IV (intravenous) cyclophosphamide and IV Topotecan and subsequently competed cycle 2 , after which tandem autologous stem cell transplants were performed at Medical University of South Carolina with a pre-transplant Curie score of 2. He then underwent primary tumor resection, subsequently completed immunotherapy following COG protocol ANBL0032, and finally protocol NMTRC-014, the Neuroblastoma Maintenance Therapy Trial using Diflouromethylornithine (DFMO). Three years after the first hospital admission, the patient returned with a left distal tibia fracture sustained after a fall from a porch. He was seen by orthopedics on call, splinted, and referred to the orthopedic clinic. This tibial fracture was slow to heal and concerning for a pathological fracture. A bone scan on the left tibia was ordered that confirmed this suspicion and a bone biopsy was conducted that confirmed neuroblastoma recurrence. Subsequent bilateral bone marrow biopsies showed no additional sites of disease. Oncology was reconsulted; DFMO protocol was discontinued, and the patient was started on COG protocol ANBL1221 with Irinotecan, Temodar, and Unituxan.

Four months later, the patient returned to the ER for leg pain and fevers and orthopedic surgery was consulted. WBC count was within normal levels (7.4x109/L), but ESR levels were highly elevated $(120 \mathrm{~mm} / \mathrm{h})$, and CRP was highly elevated $(15.2 \mathrm{mg} / \mathrm{L})$. Chemotherapy was postponed due to thrombocytopenia. The patient was started on another round of empiric antibiotic coverage due to meeting sepsis criteria. Plain radiographs demonstrated a lytic lesion at the level of the left distal tibia (Figure 1 \& 2). A sedated MRI was obtained with T2-weighted images suggestive of a pyogenic collection and reactive edema at the level of the distal tibia consistent with osteomyelitis. Two subsequent biopsy samples were obtained in sterile fashion that did not reveal any organisms but did show increased neutrophils. The wound was then irrigated copiously, and the skin was closed with chromic suture (Figure 3).

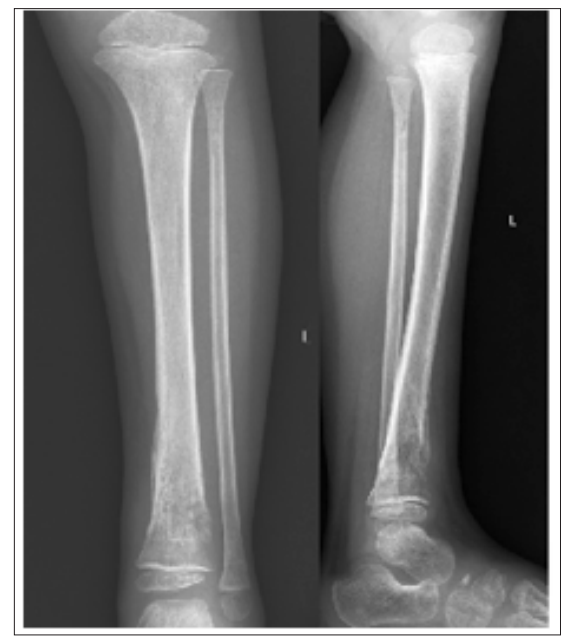

Figure 1: Site of left distal tibia lytic lesion.

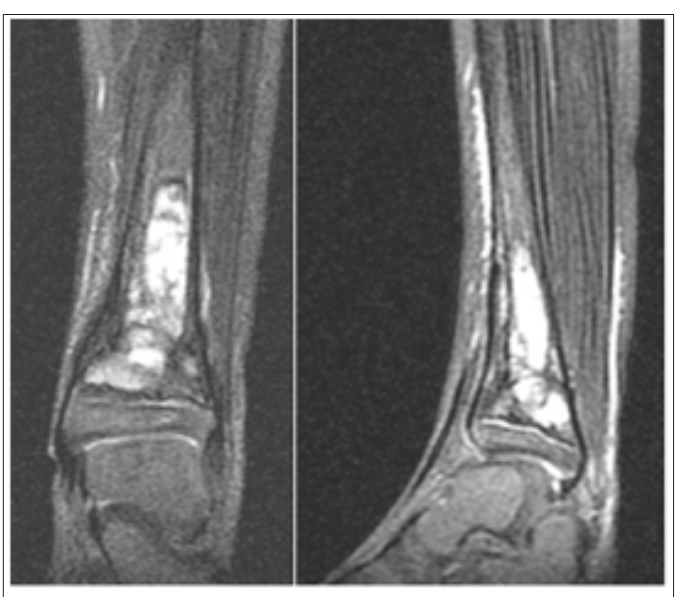

Figure 2: MRI T2W demonstrating edema and serpiginous enhancement suggestive of osteomyelitis.

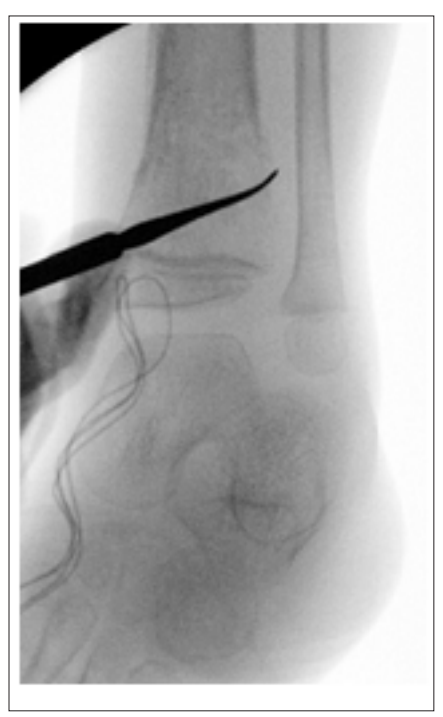

Figure 3: Biopsy site left distal tibia. 


\section{Conclusion}

To the best of our knowledge, this case illustrates the first reported incidence of neuroblastoma malignancy recurrence three years after remission with associated osteomyelitis at the same site. Throughout our patient's hospital course, every effort was taken in order to ensure a sterile working field when managing his condition. Following standard sterile technique, there was minimal risk of iatrogenic contamination to the distal tibia when performing bone biopsies to evaluate his pathological fracture. In addition, his empiric antibiotic coverage would have provided further prophylaxis. His extensive chemotherapy regimen would have played a role in immunosuppression and the patient's predisposition to subsequent infection. However, for the aforementioned reasons of good sterile technique and antibiotic coverage, osteomyelitis is a particularly unlikely and unexpected infection in this patient. Taking all these factors into account, this case provides fascinating insight into how oncological pathologies could be associated with the manifestation of independent infectious etiologies. Previously reported cases of comorbidities with neuroblastoma have focused largely on genetic conditions with minimal reports of infectious comorbidities. As high-risk neuroblastomas have one of the lowest survival rates of all childhood cancers, it remains critical to be vigilant of any conditions that the malignancy predisposes, including infection, so that treatment can be optimized and directed effectively.

\section{Statement of Ethics}

Informed consent was obtained by patient for publication of this case and all related images.

\section{Disclosure Statement}

The authors have no conflicts of interest to declare.

\section{Funding Sources}

No specific funding was received for this study.

\section{Author Contributions}

Vamsi Reddy, Clive Miranda, and Benjamin Sookhoo participated in the production of the manuscript literature. Nitish Sood assisted with grammatical corrections and final production. David Cearley is the attending physician that performed the final review of completed document.

\section{References}

1. Heck JE, Ritz B, Hung RJ, Hashibe M, Boffetta P, et al. (2009) The epidemiology of neuroblastoma: A review. Paediatric and Perinatal Epidemiology 23(2): 125-143.

2. Dumba M, Jawad N, McHugh K (2015) Neuroblastoma and nephroblastoma: A radiological review. Cancer Imaging 15(1): 5-5.

3. Ora I, Eggert A (2011) Progress in treatment and risk stratification of neuroblastoma: Impact on future clinical and basic research. Seminars in Cancer Biology 21(4): 217-228.

4. Survival rates for neuroblastoma.

5. Perez CA, Matthay KK, Atkinson JB, Seeger RC, Shimada H, et al. (2000) Biologic variables in the outcome of stages I and II neuroblastoma treated with surgery as primary therapy: A children's cancer group study. Journal of Clinical Oncology 18(1): 18-26.

6. Schmidt ML, Lukens JN, Seeger RC (2000) Biologic factors determine prognosis in infants with stage IV neuroblastoma: A prospective Children's Cancer Group study. Journal of Clinical Oncology 18(6): 12601268.

7. Conte M, Parodi S, De Bernardi B (2006) Neuroblastoma in adolescents: The italian experience. Cancer 106(6): 1409-1417.

8. Kaneko Y, Kobayashi H, Watanabe N, Tomioka N, Nakagawara A, et al. (2006) Biology of neuroblastomas that were found by mass screening at 6 months of age in Japan. Pediatric Blood \& Cancer 46(3): 285-291.

9. Brodeur GM, Look AT, Shimada H (2001) Biological aspects of neuroblastomas identified by mass screening in Quebec. Medical and Pediatric Oncology 36(1): 157-159.

10. Lonergan GJ, Schwab CM, Suarez ES, Carlson CL (2002) Neuroblastoma, ganglioneuroblastoma, and ganglioneuroma: Radiologic-pathologic correlation. Radiographics 22(4): 911-934.

11. Capasso M, Diskin SJ (2010) Genetics and genomics of neuroblastoma. Cancer Treatment and Research 155: 65-84.

For possible submissions Click below: 